Dementia

and Geriatric

Cognitive Disorders
Dement Geriatr Cogn Disord 2006;21:309-315

DOI: $\underline{10.1159 / 000091522}$
Accepted: October 18, 2005

Published online: February 14, 2006

\title{
Cognitive Profiles in Alzheimer's Disease and in Mild Cognitive Impairment of Different Etiologies
}

\author{
David A. Loewenstein ${ }^{a, b, d}$ Amarilis Acevedo ${ }^{b, d, e}$ \\ Joscelyn Agron $^{b, d, e}$ Richard Issacson $^{a}$ Silvia Strauman ${ }^{a, b, d}$ \\ Elizabeth Crocco $^{\text {b,d }}$ Warren W. Barker ${ }^{a}$ Ranjan Duara ${ }^{a, c}$ \\ ${ }^{a}$ Wein Center for Alzheimer's Disease and Memory Disorders, Mount Sinai Medical Center, Miami Beach, Fla., \\ ${ }^{\mathrm{b}}$ Center on Aging and Departments of ${ }^{\mathrm{c}}$ Medicine and ${ }^{\mathrm{d}}$ Psychiatry and Behavioral Sciences, Miller School of \\ Medicine, University of Miami, Miami, Fla., e Johnnie Byrd Alzheimer's Disease Clinical and Research Center, \\ Tampa, Fla., USA
}

\section{Key Words}

Mild cognitive impairment $\cdot$ Mild cognitive impairment vascular subtypes · Alzheimer's disease

\begin{abstract}
There has been increasing interest in determining whether amnestic, nonamnestic and multiple-domain subtypes of mild cognitive impairment (MCl) reflect different disease etiologies. In this study, we examined the extent to which cognitive profiles of nondemented patients with $\mathrm{MCl}$ diagnosed with prodromal Alzheimer's disease (AD) differed from those $\mathrm{MCl}$ patients diagnosed with vascular disease. We also compared these diagnostic groups to mildly demented patients diagnosed with $A D$ and normal elderly controls. Results indicate that a majority of both $\mathrm{MCl}-\mathrm{AD}$ and $\mathrm{MCl}$-vascular patients experienced amnestic features and that multiple-domain was the most common presentation. MCl-AD and $\mathrm{MCl}$-vascular groups did not differ on neuropsychological measures tapping memory, language, visuospatial skills/ praxis or executive function. Further both $\mathrm{MCl}$ groups could be distinguished from dementia patients with re-
\end{abstract}

gards to performance on measures of memory but not on non-memory measures. Considerable variability was observed in the degree of memory impairment among $\mathrm{MCl}$ patients with scores as much as 6 standard deviations below expected mean values. $\mathrm{MCl}-\mathrm{AD}$ and $\mathrm{MCl}-$ vascular patients frequently exhibit both common and overlapping amnestic and nonamnestic features. The implication of these findings for future clinical research is discussed.

Copyright $(2006$ S. Karger AG, Basel

\section{Introduction}

Mild cognitive impairment (MCI) is an intermediate state between normal cognition and dementia. Although MCI differs from a normal cognitive state by the presence of cognitive impairment, there is not sufficient impairment in functional status to meet criteria for a dementia syndrome. MCI can be classified into several cognitively defined subtypes, including amnestic, nonamnestic and multi-domain MCI [1, 2]. A major impetus for this classification is to determine whether an association exists

\section{KARGER}

(c) 2006 S. Karger AG, Basel

Fax +41613061234 E-Mailkarger@karger.ch www.karger.com www.karger.com/dem
David Loewenstein, $\mathrm{PhD}$

Department of Psychiatry and Behavioral Sciences, University of Miami, 2nd Floor MRI Building, Mount Sinai Medical Center, 4300 Alton Road

Miami Beach, FL 33140 (USA)

Tel. +1 305674 2194, Fax +1 305532 5241, E-Mail dloewenstein@worldnet.att.net 
between the cognitive subtype of MCI and its etiology, (e.g. prodromal Alzheimer's disease (AD), vascular disease or other etiologies) and the prognosis of the MCI syndrome (i.e., rate of progression to dementia, reversibility or prolonged stability). Subjects recruited from memory disorder clinics, who meet criteria for the amnestic subtype of MCI, have been found to progress to $\mathrm{AD}$ at a rate of $12 \%$ per year [3] and from 20 to $50 \%$ over a 3-year period [4-6]. Indeed, it has been reported that delayed recall, rate of forgetting and other deficits in episodic memory may be indicative of incipient or early AD $[7,8]$. In other studies, those cases classified as nonamnestic MCI may have a primary vascular etiology [3].

Results from the Cardiovascular Aging and Health Study [9] indicate that subjects with infarctions on MRI were at greater risk for amnestic MCI. Most of the MCI subjects in that study had impairments in multiple cognitive domains. More recently, Rasquin et al. [10] found that multiple domain MCI was sensitive to identifying persons at high risk for a subsequent diagnosis of both $\mathrm{AD}$ and vascular dementia. Our group has also reported that memory impairment, as measured by a test of object memory learning, is a better predictor than age, ApoE genotype or other neuropsychological variables in the prediction of rate of conversion to dementia among MCI patients diagnosed with either an Alzheimer's or vascular etiology [11]. While vascular cognitive impairment has been associated with predominant attentional and executive impairment [12], a recent comprehensive review of the literature by Backman et al. [2] concluded that preclinical AD subjects can be discriminated from normal elderly controls on a wide array of measures, including those measuring perceptual speed, visuospatial abilities, executive function and attention. It is, therefore, currently unknown whether MCI subjects can be differentiated on the basis of predominant memory or other neuropsychological deficits into MCI-vascular or MCI-AD etiological subtypes.

\section{Methods}

\section{Subjects}

We recruited subjects for this study from among those individuals who presented with a variety of cognitive and noncognitive complaints to the Wien Center for Alzheimer's Disease and Memory Disorders, Miami Beach. Many subjects, or their informants, listed memory impairment as their principal complaint, but the major presenting symptoms also included language deficits, changes in personality or judgment, psychiatric symptoms or changes in gait and balance or other motor symptoms. One-hundred and twenty-two individuals were initially recruited into the present study.
Seventy-two of these persons received an evaluation for cognitive symptoms, including a neurological history and examination, blood tests and an MRI scan of the brain. The diagnosis of MCI was made on the basis of this clinical evaluation, without any influence from the neuropsychological evaluation results. The clinical criteria for MCI and its etiological subtypes have been employed in studies at this Center previously, and are similar to clinical criteria, described for the diagnosis of cognitive impairment, no dementia (CIND) [11].

Briefly, the diagnosis of MCI required a cognitive complaint from the subject or an informant, the absence of dementia, as determined by DSM-IV criteria for dementia [13], and the presence of cognitive deficits on behavioral neurological examination in domains that include orientation, recent memory (word list recall), remote memory (autobiographical memory), language (naming and comprehension), praxis, visuospatial function, judgment and problem solving (calculations) and comportment (appropriateness, attentiveness, motivation and lability of affect). Duration of cognitive deficits (from the history) and the presence or absence of progression of the cognitive deficits were not material to the diagnosis of MCI.

\section{Mild Cognitive Impairment-Alzheimer's Disease}

Thirty-six subjects (17 males and 19 females) in this group fulfilled the criteria for MCI described above, and had a gradually progressive course of cognitive impairment, with predominantly memory-related symptoms. They had a global Clinical Dementia Rating Scale (CDR) [14] score of 0.5. The behavioral neurological examination revealed deficits in memory, with or without other cognitive deficits. The comportment of the subjects did not deviate from normal social behavior, with the possible exception of mild apathy and reduced motivation. The general neurological examination revealed no focal signs suggestive of cerebral infarction. In addition, MRI scans were assessed for features thought to be consistent with a diagnosis of incipient AD (i.e., evidence of cortical, hippocampal and entorhinal cortex atrophy, ventricular enlargement, and absence of significant cortical or subcortical infarct load). The presence of periventricular or other white matter hyperintensities on MRI were not considered material to the diagnosis of MCI$\mathrm{AD}$, as long as cerebral infarcts were not present. These individuals met NINCDS-ADRDA [15] clinical criteria for probable AD, with the exception that there was not sufficient impairment in social/occupational function to merit a diagnosis of dementia. Previous research has indicated that persons diagnosed in this manner have prodromal $\mathrm{AD}$ and that all convert to dementia over a 9.5-year period [16].

\section{MCI-Vascular}

Twenty-one subjects (14 males and 7 females) in this group fulfilled the criteria for MCI described above and had a global CDR score of 0.5 . These patients and/or their informants typically reported memory concerns. Apathy, depression, irritability and emotional lability were frequent observations in the history. Abrupt onset and/or a step-wise progression were present frequently, but were not required. Prominent vascular risk factors were invariably present, including a history of stroke, 'ministroke' or 'TIA', hypertension and elevated blood pressure on examination, diabetes mellitus, hyperlipidemia, arteriosclerotic heart disease, atrial fibrillation, peripheral vascular disease, cigarette smoking and a family history of vascular disease. Behavioral neurological evaluation re- 
Table 1. Demographic characteristics and neuropsychological scores (mean \pm SD) for study groups

\begin{tabular}{|c|c|c|c|c|c|}
\hline & $\begin{array}{l}\text { MCI-AD } \\
(\mathrm{n}=36)\end{array}$ & $\begin{array}{l}\text { MCI-vascular } \\
(\mathrm{n}=21)\end{array}$ & $\begin{array}{l}\text { Mild AD } \\
(n=15)\end{array}$ & $\begin{array}{l}\text { Normal } \\
(\mathrm{n}=50)\end{array}$ & $\begin{array}{l}\text { F or } \chi^{2} \\
\text { value }\end{array}$ \\
\hline Age, years & $80.2 \pm 6.0$ & $79.7 \pm 7.0$ & $78.9 \pm 5.5$ & $79.4 \pm 4.0$ & 0.26 \\
\hline Education, years & $11.8 \pm 3.9^{\mathrm{a}}$ & $14.7 \pm 4.6^{\mathrm{a}}$ & $13.3 \pm 3.6^{\mathrm{a}}$ & $14.4 \pm 4.0^{\mathrm{a}}$ & $3.62 *$ \\
\hline Females, $\%$ & 52.8 & 33.3 & 53.3 & 60.0 & $\begin{array}{l}\chi^{2}=4.23 \\
p>0.23\end{array}$ \\
\hline MMSE & $25.54 \pm 2.1^{\mathrm{b}}$ & $27.1 \pm 1.9^{\mathrm{c}}$ & $22.9 \pm 2.8^{\mathrm{a}}$ & $27.7 \pm 1.6^{\mathrm{c}}$ & $26.45^{* * *}$ \\
\hline 3-Trial OME & $16.6 \pm 4.3^{\mathrm{b}}$ & $19.1 \pm 4.0^{\mathrm{b}}$ & $11.1 \pm 5.0^{\mathrm{a}}$ & $25.0 \pm 2.3^{\mathrm{c}}$ & $71.87 * * *$ \\
\hline Logical Memory immediate & $26.7 \pm 11.1^{\mathrm{b}}$ & $29.5 \pm 11.0^{\mathrm{b}}$ & $16.4 \pm 9.5^{\mathrm{a}}$ & $41.0 \pm 9.5^{\mathrm{c}}$ & $26.40 * * *$ \\
\hline Logical Memory delay & $12.8 \pm 8.9^{\mathrm{b}}$ & $15.7 \pm 8.7^{\mathrm{b}}$ & $5.0 \pm 3.6^{\mathrm{a}}$ & $25.8 \pm 7.9^{\mathrm{c}}$ & $32.61 * * *$ \\
\hline Trails B & $194.9 \pm 83.2^{\mathrm{b}, \mathrm{c}}$ & $161.5 \pm 89.0^{\mathrm{a}, \mathrm{b}}$ & $198.3 \pm 90.8^{b}$ & $111.0 \pm 43.2^{\mathrm{a}}$ & $11.63^{* * *}$ \\
\hline Block Design WAIS-III & $21.5 \pm 7.4^{\mathrm{a}}$ & $25.9 \pm 8.9^{\mathrm{a}, \mathrm{b}}$ & $22.3 \pm 9.2^{\mathrm{a}}$ & $29.6 \pm 8.4^{\mathrm{b}}$ & $7.54 * * *$ \\
\hline FAS Letter Fluency & $29.7 \pm 12.0^{\mathrm{a}}$ & $27.1 \pm 14.0^{\mathrm{a}}$ & $29.9 \pm 10.3^{\mathrm{a}}$ & $39.8 \pm 9.5^{\mathrm{b}}$ & $9.40 * * *$ \\
\hline Category Fluency & $29.1 \pm 9.1^{\mathrm{a}}$ & $31.0 \pm 11.1^{\mathrm{a}}$ & $28.6 \pm 9.5^{\mathrm{a}}$ & $45.5 \pm 9.0^{\mathrm{b}}$ & $27.82 * * *$ \\
\hline
\end{tabular}

Means within a given row with different superscripts are statistically significant at $\mathrm{p} \leq 0.05$ by Scheffé procedure. $* \mathrm{p}<0.05 ; * * \mathrm{p}<0.01 ; * * * \mathrm{p} \leq 0.001$.

vealed deficits in memory or other cognitive domains. Apathy, depression or an emotionally disinhibited and labile state were frequently observed. These individuals had focal findings on neurological examination as well as findings on MRI brain scans that included multiple cortical or, more frequently, subcortical lacunar infarcts, often associated with extensive white matter hyperintensities in the periventricular and subcortical white matter regions. Hippocampal and entorhinal cortex atrophy on $1.5-\mathrm{mm}$-thick $\mathrm{T}_{1^{-}}$ weighted coronal slices was rated as mild, minimal or no atrophy. These criteria are consistent with, but more rigorous than those for vascular CIND [17].

\section{Mild AD}

Fifteen subjects ( 7 males and 8 females) in this group or their informants reported memory concerns in the patient, with memory changes reported as insidious in onset and gradual in progression. They had a global CDR score of 1.0 and MRI scans showed features consistent with a diagnosis of $\mathrm{AD}$ (i.e., the MRI features described above, under MCI-AD). These individuals met NINCDSADRDA clinical criteria for probable or possible AD and had sufficient impairment in social/occupational function to merit a diagnosis of dementia.

\section{Neuropsychological Test Battery}

Each patient in the study received a neuropsychological test battery administered in his/her native language (English or Spanish). The neurologist was blind to these neuropsychological test results but all patients classified as having MCI or dementia required impairment in at least one cognitive domain at $1.5 \mathrm{SD}$ or more below the mean of age- and/or education-matched normative data for English speakers and Spanish speakers to: (a) obtain objective confirmation of cognitive deficits and to (b) to allow cognitive subtype analyses as described below. Tests that were administered were as follows: Memory: Total Recall of Three Trial Fuld Object Memory Evaluation (OME) [18]; Delayed Recall for Passages (WMS-III)
[19], and Delayed Visual Reproduction (WMS-R) [20]; Language: Controlled Oral Word Association Test [21]; Visuospatial Skills: Block Design WAIS-III [22]; Executive Function: Trails B [23].

Neuropsychological classification of nondemented, nonnormal subjects into cognitive subgroups was as follows: (a) amnestic MCI: one or more memory tests $1.5 \mathrm{SD}$ or greater below expected normative values. All nonmemory tests were within normal limits; (b) nonamnestic MCI: no memory measure greater than 1.0 SD below expected normative values but one or more nonmemory measure 1.5 SD or more below expected values; (c) multiple-domain MCI: One or more memory test $1.5 \mathrm{SD}$ or greater below expected normative values and one or more nonmemory tests $1.5 \mathrm{SD}$ or below expected values.

\section{Cognitively Normal Community-Dwelling Elderly}

The cognitively normal group consisted of 50 subjects (20 males and 30 females) who were recruited from an ongoing longitudinal study of predictors of cognitive decline in normal aging or MCI. They gave no history of cognitive symptoms, and they received a CDR global score of 0 after an intensive standardized interview by trained neuropsychologists (A.A., J.A. or D.A.L.) or postdoctoral fellows in neuropsychology under their direct supervision. All of these subjects scored within normal limits on a neuropsychological battery of tests tapping memory, language, visuospatial skills/praxis and executive function, as described above.

\section{Results}

As indicated in table 1, there were no significant differences between groups with regards to age $[F(3,118)=$ $0.26 ; \mathrm{p}=0.85]$. Although there were initial indications of educational differences using ANOVA $[\mathrm{F}(3,117)=3.62$; 
Table 2. Standard deviations of below age and education appropriate normative values for each diagnostic group on the 3-Trial OME

\begin{tabular}{lcccccc}
\hline & Normal & $1.5-2.0$ SD & $2.5-3.0$ SD & $3.5-4.0$ SD & $4.5-5.0$ SD & $5.5+$ SD \\
\hline MCI-AD, \% & 22.2 & 13.9 & 22.2 & 22.2 & 8.3 & 11.1 \\
MCI-vascular, \% & 33.3 & 28.6 & 9.5 & 23.8 & 4.8 & 0 \\
Mild AD, \% & 0 & 0 & 13.3 & 26.7 & 33.3 & 26.7 \\
\hline
\end{tabular}

$\chi^{2}=23.10($ d.f. $=10) ; \mathrm{p} \leq 0.01$.

$p<0.02$ ], post-hoc tests of means using the Scheffe' procedure did not reveal any statistically significant differences. Similarly, there were no group differences with regards to the proportions of males $(47.5 \%)$ vs. females $(52.5 \%)$ in any of the study groups $\left[\chi^{2}(\right.$ d.f. $=3)=4.23$; $\mathrm{p}=0.24]$ or those tested in English $(68.0 \%)$ vs. Spanish $(32.0 \%)\left[\chi^{2}(\right.$ d.f. $\left.=3)=1.38 ; p=0.71\right]$. Mild AD patients had the lowest MMSE scores followed by MCI-AD patients. The highest MMSE scores were obtained by MCIvascular patients and normal elderly controls.

An analysis of table 1 reveals that on a majority of memory measures, both MCI-AD and MCI-vascular had statistically equivalent scores that were higher than those in the mild AD group. Normal elderly controls had higher scores on these measures than all of the other study groups. Further, MCI-AD, MCI-vascular and mild AD groups evidenced statistically equivalent scores that were lower than those of normal elderly on nonmemory measures including measures such as the FAS Controlled Oral Word Association Test and Category Fluency. MCI-AD patients had lower scores than normal elderly on all measures.

MCI-AD, MCI-vascular and mild AD patients were classified as different cognitive subtypes (amnestic, nonamnestic or multiple domain) based solely on their neuropsychological test performance. Chi-square analyses $\left[\chi^{2}(\right.$ d.f. $\left.=4)=7.14 ; p=0.13\right]$ did not indicate statistically significant differences in proportions of these cognitive subgroups among our study groups. MCI-AD and MCI-vascular groups had a purely amnestic form of MCI (22.2 and $19.0 \%$, respectively) compared to $46.7 \%$ of mild AD patients. Multiple-domain MCI was the predominant diagnosis for all of the study groups with $58.3 \%$ of MCIAD patients, $52.4 \%$ of MCI-vascular patients, and 53.3\% of mild $\mathrm{AD}$ patients evidencing this particular subtype.

We expressed the degree of memory impairment for each of the MCI and dementia groups by calculating the number of standard deviations below the mean each patient scored on the 3-Trial Fuld OME as compared to age, education and culturally appropriate normative data, as described by Loewenstein et al. [18]. For this particular analysis we avoided exaggerating the differences between the MCI, dementia and normal subjects by using previously published norms rather than normative data from the current study. This was important given that normal subjects in the current study were selected on the basis of having a normal or above normal Fuld OME score. As indicated in table 2, the criterion used to classify subjects as cognitively normal was a score that was no greater than 1.0 SD below the expected mean on any test; mildly impaired subjects scored 1.5-2.0 SD below the expected mean; moderately impaired subjects scored 2.5-3.0 SD below the expected mean and moderately severe impaired subjects scored 3.5-4.0 SD below the expected mean. The most severely impaired patients were 4.5-5.5 SD or more below the expected mean. The $\chi^{2}$ value $[23.10($ d.f. $=10)$; $\mathrm{p} \leq 0.001]$ indicated a statistically significant difference in the proportions of subjects within each category.

The median number of SD below the mean for the MCI-AD groups was 2.5, with a range of $2 \mathrm{SD}$ above the expected mean to $6.0 \mathrm{SD}$ below the expected mean. In contrast, the median SD below the expected mean for the MCI-vascular group was 1.5, with a range of $1.5 \mathrm{SD}$ above the expected mean to 5.0 SD below the expected mean.

\section{Discussion}

One major goal of the current study was to determine the extent to which nondemented subjects diagnosed as MCI-AD vs. MCI-vascular exhibited different cognitive subtypes and whether and how these groups would differ neuropsychologically from AD patients diagnosed with mild dementia. In our sample of patients, there was no statistical difference in the proportion of cognitive subtypes among MCI and dementia groups. Multiple-domain impairment (amnestic plus nonamnestic impair- 
ment) was the most common form of presentation among dementia and MCI subjects, consistent with previous studies $[1,2]$. Some form of amnestic impairment was found among the majority of MCI-AD (80.5\%) and MCIvascular (71.4\%) patients.

The two MCI groups did not differ with regard to performance on neuropsychological measures tapping memory and other domains. Measures of delayed recall and decay of information upon delay have been suggested as markers of early $\mathrm{AD}[7,8]$, while difficulties with attention, executive function and motor speed may be associated with cerebrovascular disorders [24]. The finding that mild AD patients scored lower than those in the MCI-AD and MCI-vascular groups is consistent with a large body of research [25]. MCI-AD patients also scored lower than normal elderly controls on language, speed of processing, visuospatial skills and executive function, which is consistent with the notion that skills other than memory are impaired in preclinical AD [2].

In this study, a wide range of variability in memory performance on the 3-Trial OME was found. For MCIAD patients, the median (as well as modal) 3-Trial OME score was $2.5 \mathrm{SD}$ below the mean, with a range of $2 \mathrm{SD}$ above the mean to $6 \mathrm{SD}$ below the mean. In contrast, the median for the MCI-vascular group was $1.5 \mathrm{SD}$ below the mean, with a range of $1.5 \mathrm{SD}$ above the mean to $5 \mathrm{SD}$ below the mean. No mild AD patient scored better than 3.0 SD below the mean on the 3-Trial Fuld OME and there was a large degree of overlap in scores between mild $\mathrm{AD}$ and MCI-AD. One third of MCI-vascular subjects and about one fifth of MCI-AD scored in the normal range on the Fuld OME. It has been previously shown that the degree of memory impairment on the baseline evaluation in cases presenting with $\mathrm{MCI}$ is predictive of the future rate of progression of cognitive deficits towards a dementia diagnosis regardless of the suspected etiology of cognitive impairment [11]. The results in this study do speak to the issue of a possible lower limit (i.e., the SD below the mean on memory tests) for distinguishing MCI from dementia and possibly even MCI-AD from MCIvascular. However, most memory measures do not provide a sufficient range of values below the mean values to allow these distinctions to be made. The 3-Trial Fuld OME, though vastly better than other traditional memory measures in distinguishing between MCI-AD and mild $\mathrm{AD}$, does not provide sufficient separation between the MCI-AD and MCI vascular subtypes to be a useful clinical tool for this purpose. Nevertheless, the findings in this study do provide the impetus for exploring the utility of other experimental memory measures that may prove useful in more accurately distinguishing MCI from dementia and the different subtypes of MCI from one another.

As is evident from the results of this study, there is considerable heterogeneity and overlap among MCI-AD, MCI-vascular and mild dementia subjects with regards to performance on tests tapping amnestic and nonamnestic domains of cognition. From a clinical perspective, it is practical and appropriate to use the presence of functional impairment as the marker of MCI to dementia progression. However, from a clinical and research perspective it is unlikely that MCI subjects who do not have functional impairment are pathophysiologically different from those who do have functional impairment and therefore meet clinical criteria for dementia.

The use of functional impairment as a surrogate for dementia, implicitly a neuropathologically worse condition than MCI, is likely to introduce several errors in research studies. The point in a progressive disease process at which functional ability begins to be impaired is dependent on a given individual's premorbid functional ability, which is highly variable and is dependent on life experiences and a number of other factors which could be lumped together in the term 'functional reserve capacity'. The determination of functional impairment remains dependent on input from an informant whose report may be influenced by various factors (e.g., longstanding biases regarding the aging process, quality of the relationship with the patient). A more appropriate and standardizable research measure of 'dementia' may be the presence and degree of memory impairment, as measured by a standardized test. The use of the cut-off of 1.5 SD below the normative mean on memory measures for distinguishing MCI from normal cognition has come into widespread use and has been shown to be useful, even though this cut-off point is arbitrary. Similarly, a cut-off of 2.5-3.0 SD below appropriate normative means on more sensitive memory measures such as the 3-Trial Fuld OME or other list learning paradigms may prove to be a useful, standardizable and more reproducible research tool for distinguishing MCI from early dementia. Our previous research has also indicated that the severity of memory impairment at baseline predicts the rate of progression to dementia [11].

Strengths of the present investigation include a clinical diagnosis of MCI-AD, MCI-vascular and mild AD that was rendered through stringent operational criteria. The only requirement for inclusion into the study for these patients was objective confirmation of at least one neuropsychological deficit. However, potential limitations in- 
clude reliance on a modest clinical sample and the possibility that additional neuropsychological measures may have identified more cases of multiple-domain MCI.

As previously described, MCI-vascular required only cognitive impairment and not necessarily an amnestic component, which is analogous to CIND criteria [12]. As neuropathological data on these subjects are lacking, it is impossible to know to what extent the clinical diagnosis was inaccurate for either MCI group. However, we believe that the MCI-vascular cases reported in this study are far more likely to have had primarily vascular pathology underlying their cognitive deficits for the following reasons: First, vascular cases were carefully worked up by an experienced behavioral neurologist who used criteria stricter than those used for vascular CIND [17], including focal neuropsychological signs and symptoms and MRI evidence of cerebral infarction and mild, minimal or no atrophy. Only those subjects with above-average infarct loads and mild or minimal hippocampal/entorhinal cortex atrophy were diagnosed to have MCI-vascular. Subjects with few infarcts but moderate or severe atrophy of the hippocampus and/or entorhinal cortex were diagnosed to have MCI-AD. In addition, over a quarter of MCI-vascular cases did not evidence memory impairment on neuropsychological testing. Finally, we evalu- ated 'conversion rates' to dementia in a previous study of MCI-AD and MCI-vascular cases, using the same clinical and neuroimaging diagnostic criteria as in this study to classify etiological subtypes of MCI. We found that the rate of conversion for MCI-vascular patients averaged half that of MCI-AD patients over an average of a 2.5year period [11]. This suggests that the criteria we have used for making the diagnosis of MCI-AD and MCI-vascular have validity, because these two subtypes of MCI had different trajectories of decline over time. In another study of prospectively diagnosed cases of vascular dementia, using criteria that were less stringent than ours, only one third of the cases was found to have AD pathology on autopsy [26].

Further work in this area will involve the follow-up of these and other MCI cases with different presumed etiologies and the examination of individual trajectories over time as well as neuropathological confirmation.

\section{Acknowledgement}

This research was supported by grant 5R01AG020094-03, (D.A.L, principal investigator) and 1P50AG025711-01 from the National Institute on Aging.

\section{References}

1 Lopez O, Jagust WJ, DeKosky S, Becker JT, Fitzpatrick A, Dulberg C, Breitner J, Lyketsos C, Jones B, Kawas C, Carlson M, Kuller L: Prevalence and classification of mild cognitive impairment in the Cardiovascular Health Study Cognition Study. Arch Neurol 2003;60: 1385-1389.

-2 Backman L, Jones S, Berger AK, Laukka EJ, Small BJ: Multiple cognitive deficits during the transition to Alzheimer's disease. J Intern Med 2004;256:195-204.

-3 Petersen RC: Mild cognitive impairment as a diagnostic entity. J Intern Med 2004;256:183194.

$\checkmark 4$ Black SE: Can SPECT predict the future for mild cognitive impairment? Can J Neurol Sci 1999;26:4-6.

$\checkmark 5$ McKelvey R, Bergman H, Stern J, Ruch C, Zehirney $\mathrm{G}$, Chertow H: Lack of prognostic significance of SPECT abnormalities in non-demented elderly subjects with memory loss. Can J Neurol Sci 1999;26:23-28.

6 Wolf H, Grunwald M, Ecke GM, Zedlick D, Bettin S, Dannenberg C, Dietrich J, Eschrich $\mathrm{K}$, Arendt T: The prognosis of mild cognitive impairment in the elderly. J Neural Transm 1998;54(suppl):31-50.

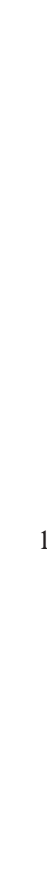

7 Locasio JJ, Growden JH, Corkin S: Cognitive Test Performance in detecting, staging and tracking Alzheimer's disease. Arch Neurol 1995;52:1087-1099.

8 Troster AI, Butters N, Salmon D, Cullum CM, Jacobs D, Brandt J, White RF: The diagnostic utility of savings scores: differentiating Alzheimer's and Huntington's disease with logical memory and visual reproduction tasks. J Clin Exp Neuropsychol 1993;5:773-788.

$\checkmark 9$ Lopez O, Jagust WJ, Dulberg C, Becker JT, DeKosky S, Fitzpatrick A, Breitner J, Lyketsos C, Jones B, Kawas C, Carlson M, Kuller L: Risk factors for mild cognitive impairment in the Cardiovascular Health Study Cognition Study. 2. Arch Neurol 2003; 60:1394-1399.

10 Rasquin S, Lodder J, Visser PJ, Lousberg R, Verhey F: Predictive accuracy of MCI subtypes for Alzheimer's disease and vascular dementia in subjects with mild cognitive impairment: a 2-year follow-up study. Dement Geriatr Cogn Disord 2005;19:113-119.
11 Luis CA, Barker WW, Loewenstein DA, Crum TA, Rogaeva E, Kawarai T, St George Hyslop P, Duara R: Conversion to dementia among two groups with cognitive impairment. A preliminary report. Dement Geriatr Cogn Disord 2004; 18:307-313.

12 Graham JE, Rockwood K, Beattie BL, McDowell I, Eastwood R, Gauthier S: Standardization of the diagnosis of dementia in the Canadian Study of Health and Aging. Neuroepidemiology 1996;15:246-256.

13 American Psychiatric Association: Diagnostic and Statistical Manual of Mental Disorders, ed 4. Washington, American Psychiatric Association, 1994.

14 Morris JC: The Clinical Dementia Rating (CDR): current versions and scoring rules. Neurology 1993;43:2412-2414.

$>15$ McKhann G, Drachman D, Folstein M, Katzman R, Price D, Stadlan EM: Clinical diagnosis of Alzheimer's disease: report of the NINCDS-ADRDA Work Group under the Auspices of Department of Health and Human Services Task Force on Alzheimer's Disease. Neurology 1984;34:939-944. 
16 Morris JC, Storandt M, Miller JP, McKeel DW, Price DL, Rubin EH, Berg L: Mild cognitive impairment represents early stage Alzheimer's disease. Arch Neurol 2001;58:397405.

$\checkmark 17$ Nyenhuis DL, Gorelick PB, Geenen EG, Smith CA, Gencheva E, Freels S, deToledo Morell L: The pattern of neuropsychological deficits in vascular cognitive impairment-no dementia (vascular CIND). Clin Neuropsychol 2004;18: 41-49.

18 Loewenstein DA, Argüelles T, Barker WW, Schram L, Ownby R, Acevedo A, Mogosky B, White G, Duara R: The utility of a modified object memory test in distinguishing between three different age groups of Alzheimer's disease patients and normal controls. J Ment Health Aging 2001;7:317-324.
19 Wechsler D: The Wechsler Memory Scale, ed 3. San Antonio, The Psychological Corporation, 1997.

20 Wechsler D: The Wechsler Memory Scale, revised. San Antonio, The Psychological Corporation, 1987.

21 Benton AL, Hamsher K: Multilingual Aphasia Examination: Manual of Instructions, ed 2. Iowa City, AJA Associates, 1989.

22 Wechsler D: The Wechsler Adult Intelligence Scale, ed 3. San Antonio, The Psychological Corporation, 1997.

23 Spreen O, Strauss E: A compendium of neuropsychological tests: administration, norms, and commentary, ed 2. New York, Oxford University Press, 1998.
24 Roman GC, Sachdev P, Royall DR, Bullock RA, Orgogoza JM, Lopez-Pouza S, Arizaga R, Wallin A: Vascular cognitive disorder: a new diagnostic category updating vascular cognitive impairment and vascular dementia. J Neurol Sci 2004; 15;226:81-87.

25 Loewenstein DA, Acevedo A, Luis CA, Crum T, Barker WW, Duara R: Semantic interference deficits and the detection of mild Alzheimer's disease and mild cognitive impairment without dementia. J Int Neuropsychol Soc 2004; 10:91-100.

26 Kalaria RN, Ballard C: Overlap between pathology of Alzheimer disease and vascular dementia. Alzheimer Dis Assoc Dis 1999;13: S115-S123. 